\title{
Search for cold gas along radio lobes in the cooling core galaxies MS0735.6+7421 and M87
}

\author{
P. Salomé ${ }^{1}$ and F. Combes ${ }^{2}$ \\ 1 Institut de Radio Astronomie Millimétrique (IRAM), Domaine Universitaire, 300 rue de la piscine, 38400 St Martin d'Hères, France \\ e-mail: salome@iram.fr \\ 2 LERMA, Observatoire de Paris, 61 av. de l'Observatoire, 75014 Paris, France
}

Received 26 May 2008 / accepted 30 June 2008

\begin{abstract}
We report CO observations towards MS0735.6+7421 a distant cooling core galaxy, and towards M87, the nearest cooling core in the center of the Virgo cluster. Both galaxies contain radio cavities that are thought to be responsible for the heating that can regulate or stop the cooling of the surrounding gas. In this feedback process, there could still be some gas cooling along filaments, along the borders of the radio cavities. Molecular gas is known to exist in clusters with cooling cores, in long and thin filaments that can be formed behind the rising bubbles inflated by the central AGN. CO emission was searched for at several locations along the radio lobes of those two galaxies, but only upper limits were found. These correspond to cold gas mass limits of a few $10^{9} M_{\odot}$ for each pointing in MS0735.6+7421, and a few $10^{6} M_{\odot}$ in M87. This non detection means that either the cooling is strongly reduced by the AGN feedback or that the gas is cooling in very localized places like thin filaments, possibly diluted in the large beam for MS0735.6+7421. For M87, the AGN heating appears to have stopped the cooling completely.
\end{abstract}

Key words. cooling flows - galaxies: elliptical and lenticular, cD - galaxies: individual: MS0735.6+7421 - galaxies: individual: M87 - cosmology: observations

\section{Introduction}

Giant ellipticals in galaxy cluster centers live in a complex environment. In some cluster cores the intra-cluster medium is known to cool down, but how much of this gas is able to fuel the central galaxy is still not clear. The hot gas is no longer thought to be flowing directly towards the central galaxy. The regulation of the intra-cluster medium (ICM) cooling involves some re-heating mechanisms like jet feedback from a central AGN and it becomes observable by the formation of X-ray emitting gas cavities. This feedback mechanism produces mass outflows, weak shocks and heating (McNamara \& Nulsen 2007), but also cooling, in long and thin filaments. Indeed, some very cold gas $(10-100 \mathrm{~K})$ has been observed in $\mathrm{CO}$ emission in many cooling core systems (Edge 2001; Salomé \& Combes 2003). High resolution imaging of $\mathrm{CO}$ showed the existence of molecular filaments in Abell 1795 (Salomé \& Combes 2004) that are confined to the borders of the radio cavities. These filaments extend even farther outward in NGC 1275 (Salomé et al. 2008). The cold gas formation may result from the interaction of the AGN with the surrounding hot gas. The filaments are thought to be cooling filaments that can fuel the giant elliptical with cooled gas. More examples of this phenomenon need to be observed to better understand the physics of the formation of the cold gas. In this work, we report a search towards two cooling core systems: MS0735.6+7421 and M87 in the Virgo cluster. These two objects are known to be the sites of very strong feedback. The cooling in M0735.6+7421 very likely occurs only in the very central region and M87 is a poor cooling core with almost no cooling, so we do not expect to find much cooled gas.

The giant galaxy MS0735.6+7421 is at a distance of $716 \mathrm{Mpc}$ (1" correponds to $3.47 \mathrm{kpc})$. In spite of its large distance, its giant $\mathrm{X}$-ray cavities are so large, inflated by radio lobes of size $550 \mathrm{kpc}$, that they are resolved by the beam of the IRAM-30 m telescope. It is then possible to probe the cold gas distribution and compare it with the X-ray, $\mathrm{H} \alpha$ and radio maps. The galaxy MS0735.6+7421 hosts a radio source $4 \mathrm{C}+74.13$. According to McNamara et al. (2005) the AGN activity could have re-heated sufficiently the ICM to stop the cooling around the central galaxy. Figure 1 shows that the radio lobe emission of $4 \mathrm{C}+74.13$ fills the X-ray cavities of the intra-cluster medium. These cavities may have displaced and compressed the hot gas surrounding the central cluster galaxy, as predicted by feedback models of cooling flows. Negative feedback models as described by Revaz et al. (2008) predict that the hot X-ray gas when compressed may eventually cool very efficiently down to very low temperatures, forming cold gas filaments visible in $\mathrm{CO}(1-0)$ emission. So in this galaxy, we searched for molecular gas emission not only in the central region but also far away from the X-ray cooling core region, along the AGN radio lobes.

M87 is the most nearby X-ray bright cooling core. It lies at a distance of $18 \mathrm{Mpc}$. X-ray mapping around M87 reveals a complex structure for the cooling gas, perturbed by several bubbles (Young et al 2002). The X-ray-cool component detected in the radio lobes is in blobs that are connected to the radio buoyant bubbles (Ghizzardi et al. 2004). In a deeper XMMNewton observation of M87, Simionescu et al. (2007) find evidence that cold, metal-rich gas is being transported outwards also outside the X-ray arms, possibly through bubble-induced mixing. Many X-ray features are better be understood if there are repetitive AGN outbursts. Depressions appear as the remnants of earlier outbursts, while shocks are seen as brightenings within the prominent X-ray arms. As in Perseus, shocks may be the most significant channel for AGN energy input into 


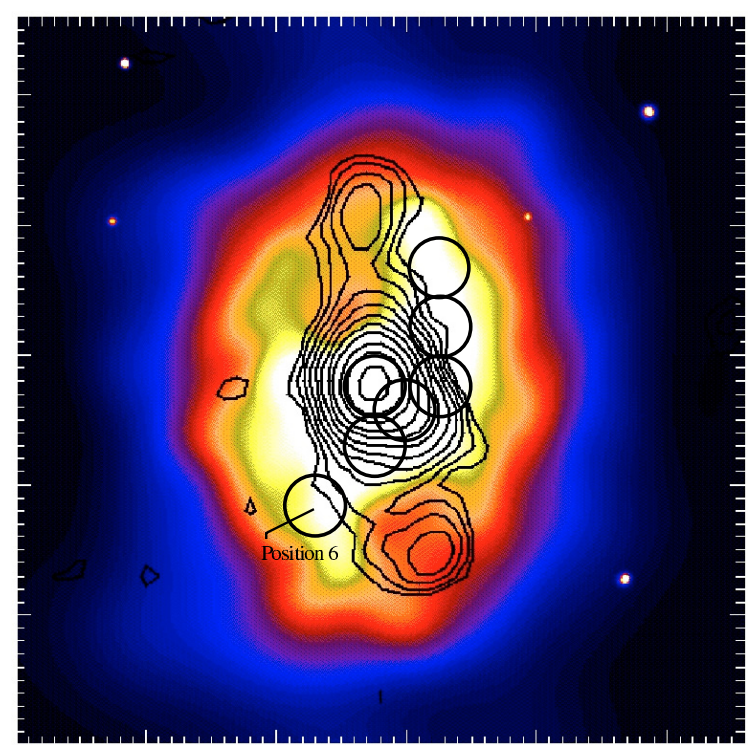

Fig. 1. A $250 \times 250^{\prime \prime}$ image of the MS0735.6+7421 cluster center (McNamara et al. 2005), one arcsec is $3.5 \mathrm{kpc}$. The $1.4 \mathrm{GHz}$ radio contours of the jets emitted by $4 \mathrm{C}+74.13$ are superposed. The cavities in the X-ray emission coincide with the radio lobe positions $(200 \mathrm{kpc}$ in size at the cluster redshift). The circles represent the $30 \mathrm{~m}$ telescope beam at $3 \mathrm{~mm}$, showing the 7 regions we observed in $\mathrm{CO}(1-0)$.

the cooling-flow atmospheres, and outbursts every $3 \times 10^{7} \mathrm{yr}$ are sufficient to quench the cooling (Forman et al. 2005).

\section{Observations}

The observations were made with the IRAM 30 m telescope on Pico Veleta, Spain during two different runs in summer 2005 and summer 2006. Weather conditions were not optimal. The first run (august 2005) suffered from wind and significant pointing corrections. The second run (may 2006) was done with clouds and $10-15 \mathrm{~mm}$ of precipitable water vapor, that means higher system temperatures. We used the wobbler switching mode with two $3 \mathrm{~mm}$ and two $1.3 \mathrm{~mm}$ receivers operating simultaneously. The beam throw was $120^{\prime \prime}$. For MS0735.6+7421, receivers were tuned to the $\mathrm{CO}(1-0)$ and $\mathrm{HCN}(2-1)$ emission lines, redshifted to the velocity of the source. The $\mathrm{CO}(2-1)$ emission line is not visible from the $30 \mathrm{~m}$ telescope at this redshift $(z=0.216)$. For M87, we observed simultaneously the CO(1$0)$ and $\mathrm{CO}(2-1)$ ines, at $3 \mathrm{~mm}$ and $1.3 \mathrm{~mm}$. Frequent pointings and focus checks were carried out using bright quasars. At $3 \mathrm{~mm}$, we used two $512 \times 1 \mathrm{MHz}$ filterbanks. This gives a total band of $\sim 1300 \mathrm{~km} \mathrm{~s}^{-1}$ for the $\mathrm{CO}(1-0)$ line setup. In addition, we used the two $250 \times 4 \mathrm{MHz}$ resolution filterbanks for the $1.3 \mathrm{~mm}$ receivers. The beams of the $30 \mathrm{~m}$ telescope, at $3 \mathrm{~mm}$ and $1.3 \mathrm{~mm}$, are $28^{\prime \prime}(94.795 \mathrm{GHz})$ and $13^{\prime \prime}(218.656 \mathrm{GHz})$ respectively for MS0735.6+7421 and 23" (114.771 GHz) and $12^{\prime \prime}(229.537 \mathrm{GHz})$ for M87.

The signals are expressed here in main beam brightness temperatures. The ratio of the antenna temperature to the main beam temperature is $T_{\mathrm{A}}^{*} / T_{\mathrm{mb}}=B_{\text {eff }} / F_{\text {eff }}$ with the ratio of the main-beam efficiency to the forward efficiency being $B_{\text {eff }} / F_{\text {eff }}=$ $0.75 / 0.95$ at $3 \mathrm{~mm}$ and $0.52 / 0.91$ at $1.3 \mathrm{~mm}$ (cf. IRAM-30 $\mathrm{m}$ site http: //www . iram.es/).

The data were calibrated with the MIRA software and reduced with the CLASS90 package. Spiky channels and bad scans were dropped and linear baselines were subtracted for each spectrum. After averaging all the spectra for each line at each
Table 1. Summary of $\mathrm{CO}(1-0)$ observations, sensitivity and emission upper limits for MS0735.6+7421.

\begin{tabular}{cccccc}
\hline \hline $\begin{array}{c}\text { Offset }(\alpha, \delta) \\
{[\operatorname{arcsec}]}\end{array}$ & $\begin{array}{c}\text { Sigma } \\
{[\mathrm{mK}]}\end{array}$ & $\begin{array}{c}I_{\mathrm{CO}} \\
{\left[\mathrm{K} \mathrm{km} \mathrm{s}^{-1}\right]}\end{array}$ & $\begin{array}{c}\text { Time } \\
{[\mathrm{min}]}\end{array}$ & $\begin{array}{c}T_{\text {sys }} \\
{[\mathrm{K}]}\end{array}$ & $\begin{array}{c}M_{\text {gas }} \\
{\left[10^{9} M_{\odot}\right]}\end{array}$ \\
\hline$(0 \times 0)$ & 0.8 & 0.24 & 90 & 137 & $\leq 12.4$ \\
$(-22 \times 0)$ & 0.9 & 0.27 & 90 & 135 & $\leq 13.9$ \\
$(-22 \times 22)$ & 0.4 & 0.12 & 210 & 146 & $\leq 6.2$ \\
$(-11 \times-11)$ & 0.8 & 0.24 & 66 & 131 & $\leq 12.4$ \\
$(0 \times-22)$ & 0.4 & 0.12 & 174 & 135 & $\leq 6.2$ \\
$(22 \times-44)$ & 0.3 & 0.09 & 573 & 189 & $\leq 4.6$ \\
$(-22 \times 44)$ & 1.9 & 0.57 & 31 & 209 & $\leq 29.3^{1}$ \\
\hline
\end{tabular}

All linewidths have been assumed to be $300 \mathrm{~km} / \mathrm{s}$. The mass upper limits are $1 \sigma$ limits. Offsets in arcsec are from the central position J2000 RA 07:41:44.5, DEC $+74: 14: 40 .{ }^{1}$ Note that the last offset was observed during only $31 \mathrm{~min}$. This explains the larger upper limit we obtain for that region.

Table 2. M87 - Summary of $\mathrm{CO}(1-0)$ and $\mathrm{CO}(2-1)$ observations: sensitivity and emission upper limits. All linewidths have been assumed at $300 \mathrm{~km} \mathrm{~s}^{-1}$. The mass upper limits are given at $1 \sigma$.

\begin{tabular}{lccccc}
\hline \hline Line & $\begin{array}{c}\text { Offset }(\alpha, \delta) \\
{[\operatorname{arcsec}]}\end{array}$ & $\begin{array}{c}\text { Sigma } \\
{[\mathrm{mK}]}\end{array}$ & $\begin{array}{c}\text { Time } \\
{[\mathrm{min}]}\end{array}$ & $\begin{array}{c}T_{\text {sys }} \\
{[\mathrm{K}]}\end{array}$ & $\begin{array}{c}M_{\text {gas }} \\
{\left[10^{6} M_{\odot}\right]}\end{array}$ \\
\hline $\mathrm{CO}(1-0)$ & $(0 \times 0)$ & 1.8 & 318 & 281 & $\leq 9.7$ \\
$\mathrm{CO}(2-1)$ & $(0 \times 0)$ & 1.8 & 318 & 680 & \\
$\mathrm{CO}(1-0)$ & $(5.5 \times 11)$ & 1.6 & 258 & 371 & $\leq 8.6$ \\
$\mathrm{CO}(2-1)$ & $(5.5 \times 11)$ & 2.2 & 258 & 1020 & \\
$\mathrm{CO}(1-0)$ & $(11 \times 0)$ & 1.7 & 361.5 & 264 & $\leq 9.2$ \\
$\mathrm{CO}(2-1)$ & $(11 \times 0)$ & 2.5 & 349.5 & 465 & \\
$\mathrm{CO}(1-0)$ & $(33 \times-22)$ & 1.1 & 260 & 330 & $\leq 5.9$ \\
$\mathrm{CO}(2-1)$ & $(33 \times-22)$ & 3.2 & 260 & 821 & \\
\hline
\end{tabular}

position, the data were Hanning smoothed. The results are summarized in Tables 1 and 2.

\section{Results}

We expected the regions where the gas can cool efficiently to be along the radio lobes and X-ray cavities, as in Abell 1795 (Salomé \& Combes 2004). This is where we looked for a past or still present cooling activity with $\mathrm{CO}$ line observations. In MS0735.6+7421, we observed 7 fields to search for $\mathrm{CO}(1-0)$ emission in the brightest $\mathrm{X}$-ray regions in the radio lobes. In M87, we observed also towards 4 offsets on the borders of the radio lobes. Only upper limits have been obtained in both galaxies.

For MS0735.6+7421 at a redshift of $z=0.216$, assuming a Hubble constant of $71 \mathrm{~km} \mathrm{~s}^{-1} \mathrm{Mpc}^{-1}$, and a flat universe with cosmological parameters $\Omega_{\mathrm{m}}=0.27$ and $\Omega_{\Lambda}=0.73$, the luminosity distance of MS0735.6+7421 is $D_{L}=1060 \mathrm{Mpc}$. The CO luminosity $L_{\mathrm{CO}}^{\prime}\left[\mathrm{K} \mathrm{km} \mathrm{s}^{-1} \mathrm{pc}^{-2}\right]$ is given by 1997 :

$$
L_{\mathrm{CO}}^{\prime}=3.25 \times 10^{7}\left[\frac{S_{\mathrm{CO}} \Delta V}{\mathrm{Jy} \mathrm{kms}}\right]\left[\frac{v_{\mathrm{obs}}}{\mathrm{GHz}}\right]^{-2}\left[\frac{D_{L}}{\mathrm{Mpc}}\right]^{2}(1+z)^{-3}
$$

We computed $\mathrm{H}_{2}$ masses by using the standard conversion factor $M_{\mathrm{H}_{2}}=4.6 L_{\mathrm{CO}}^{\prime} \quad M_{\odot}$ that assumes self-gravitating clouds. Note that this mass could be overestimated by a factor 5 if the gas is optically thin and subthermally excited (Downes \& Solomon 1998). We applied the same formula to determine the CO luminosity of M87 and assumed a distance of 


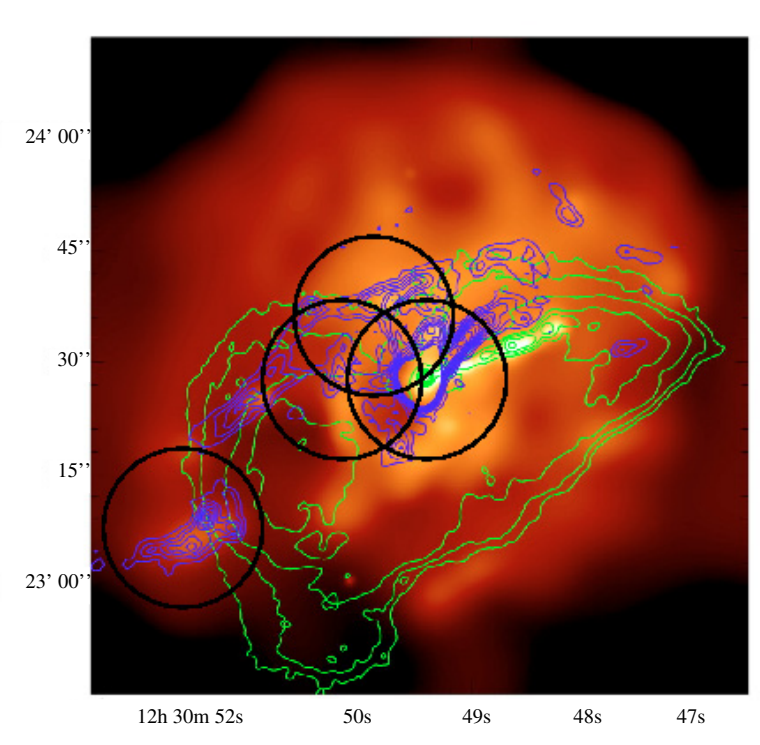

Fig. 2. M87 - X-ray image of M87 by Young et al. (2002). The white contours are the $6 \mathrm{~cm}$ radio emission and the dark contours show the $\mathrm{H}_{\alpha}$ nebulosities along the edges of the radio lobes. The circles represent the $30 \mathrm{~m}$ telescope beam size at $3 \mathrm{~mm}$.

$18 \mathrm{Mpc}(z=0.00436)$ to this source. The integrated CO intensity $S_{\mathrm{CO}}\left[\mathrm{Jy} \mathrm{km} \mathrm{s}^{-1}\right]$ is:

$S_{\mathrm{CO}}=4.95 \int T_{\mathrm{mb}}(\mathrm{CO}) \mathrm{d} V$,

where $T_{\mathrm{mb}}(\mathrm{CO})$ is the main beam brightness temperature, obtained with the CO emission line, $\mathrm{d} V$ is the line width and 4.95 the telescope $S / T_{\mathrm{mb}}$ factor in Jy per K. To express upper limits, we smoothed the spectra to $50 \mathrm{~km} \mathrm{~s}^{-1}$ resolution, and assumed a standard linewidth of $\mathrm{d} V=300 \mathrm{~km} \mathrm{~s}^{-1}$.

\subsection{MS0735. $6+7421$}

The analysis of the data does not show any evidence of $\mathrm{CO}(1-0)$ in any of the 7 positions investigated.

At the distance of MS0735.6+7421, the $\mathrm{CO}(1-0)$ spectra could be very broad (due to the mixing of many molecular clouds at different velocities) and even broader in the very central region (Salomé et al. 2008) making it very difficult to detect. Stacking the emission of all the offsets together did not improve much the signal to noise. This means that either the amount of cold gas is small, or a large velocity dispersion exists.

For MS0735.6+7421, the $28^{\prime \prime}$ field of view of the $30 \mathrm{~m}$ telescope corresponds to a diameter of $97 \mathrm{kpc}$. Recent observations of NGC 1275 (Salomé et al. 2008) have shown that the molecular gas, in cooling core galaxies, may lie in very thin and elongated filaments of at most $\sim(2 \mathrm{kpc} \times 10 \mathrm{kpc})$. If this is the case for MS0735.6+7421, it means a beam filling factor of $0.4 \%$ for such a filament. So even with a large collection of filaments, we expect a significant dilution effect in the antenna beam.

The upper limits on the amount of cold molecular gas are summarized in Table 1 . We find on average $M_{\text {gas }} \leq$ a few $10^{9} M_{\odot}$ inside a beam of diameter $97 \mathrm{kpc}$. In a multiphase cooling flow scenario, the mass deposition rate evaluated from X-rays is $260 M_{\odot} / y r$ inside $30^{\prime \prime}$ (Gitti et al. 2007). For a typical cooling time of $10^{8} \mathrm{yr}$, this means that a steady reservoir of at least $10^{10} M_{\odot}$ would exist (and even more for a star formation

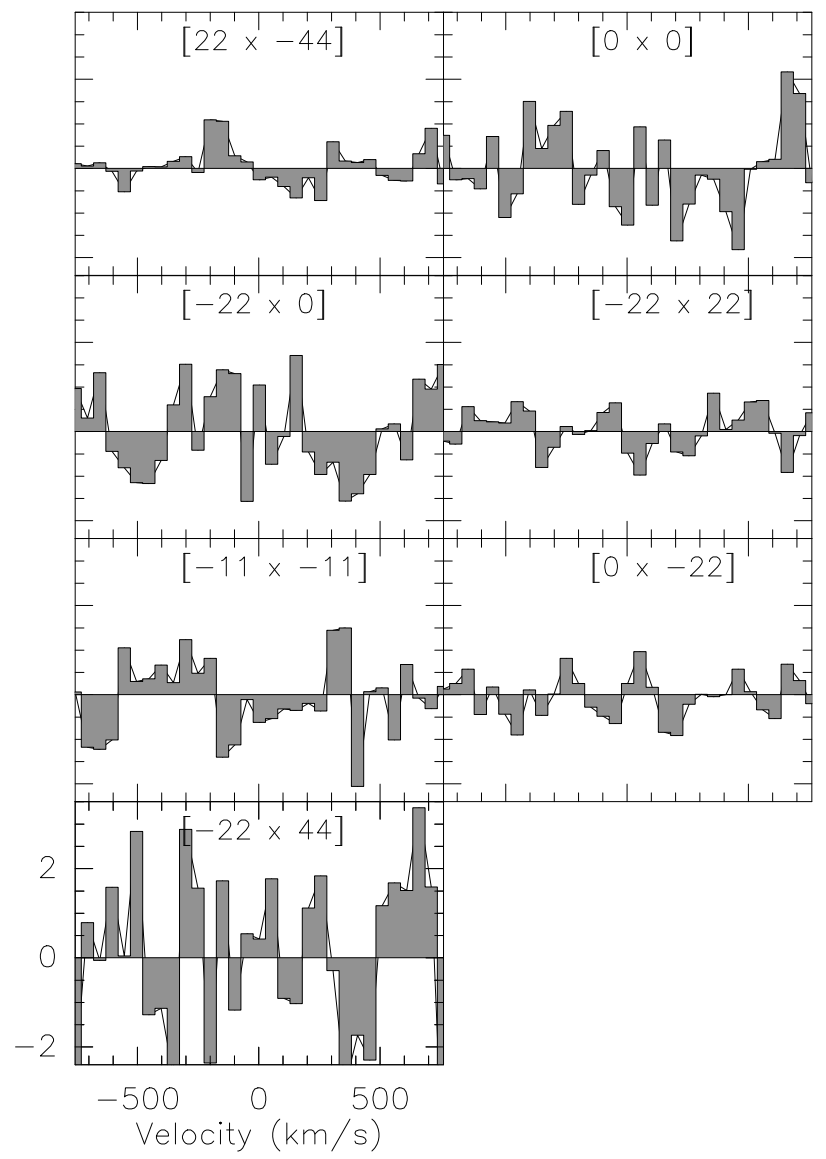

Fig. 3. MS0735.6+7421 - $\mathrm{CO}(1-0)$ spectra obtained at all the positions observed as indicated at upper right in each diagram and as overlaid on Fig. 1. The channel width is $50.6 \mathrm{~km} \mathrm{~s}^{-1}$. See Table 1 for a summary. The $Y$-axis is in $T_{\mathrm{mb}}(\mathrm{mK})$.

rate lower than the mass deposition rate). Our total upper limit, obtained by summing over all the central regions observed, is close to a few $10^{10} M_{\odot}$. Considering the uncertainties on the mass evaluation, this means that we cannot rule out that some gas is indeed cooling but not yet visible in CO.

Edge (2001) and Salomé \& Combes (2003) have shown the existence of a correlation between $\mathrm{CO}$ emission and $\mathrm{H} \alpha$ luminosity in cooling core clusters. If we refer to this relation, we can evaluate the expected molecular gas mass from the known $\mathrm{H}_{\alpha}$ luminosity coming from the central $25 \mathrm{kpc}$ (Donahue et al. 1992). This gives $\sim 6 \times 10^{10} M_{\odot}$ which is slightly larger than, but still consistent with, the $1 \sigma$ upper limits we found here (offset $[0,0]$ ). We do not find $\mathrm{CO}$ line emission either far away from the cooling cores (other offsets), where there is no optical emission line.

\section{2. $M 87$}

M87 has been identified as a very moderate cooling core (Forman et al. 2007). It is possible that the present AGN heating has completely quenched the cooling flow. Young et al. (2002) compared the optical emission with Chandra X-ray images (see Fig. 2). Filaments are observed along the edges of the radio lobes, which coincide with the sites where the X-ray gas is cooler, as in usual cooling core clusters. The filaments have a total $\mathrm{H} \alpha$ luminosity of $3 \times 10^{40} \mathrm{erg} \mathrm{s}^{-1}$ in $1250 \operatorname{arcsec}^{2}$ (Sparks et al. 1993).

Dynamical models of hot bubbles of gas injected by the active galactic nuclei (AGN), rising buoyantly into the intra-cluster 


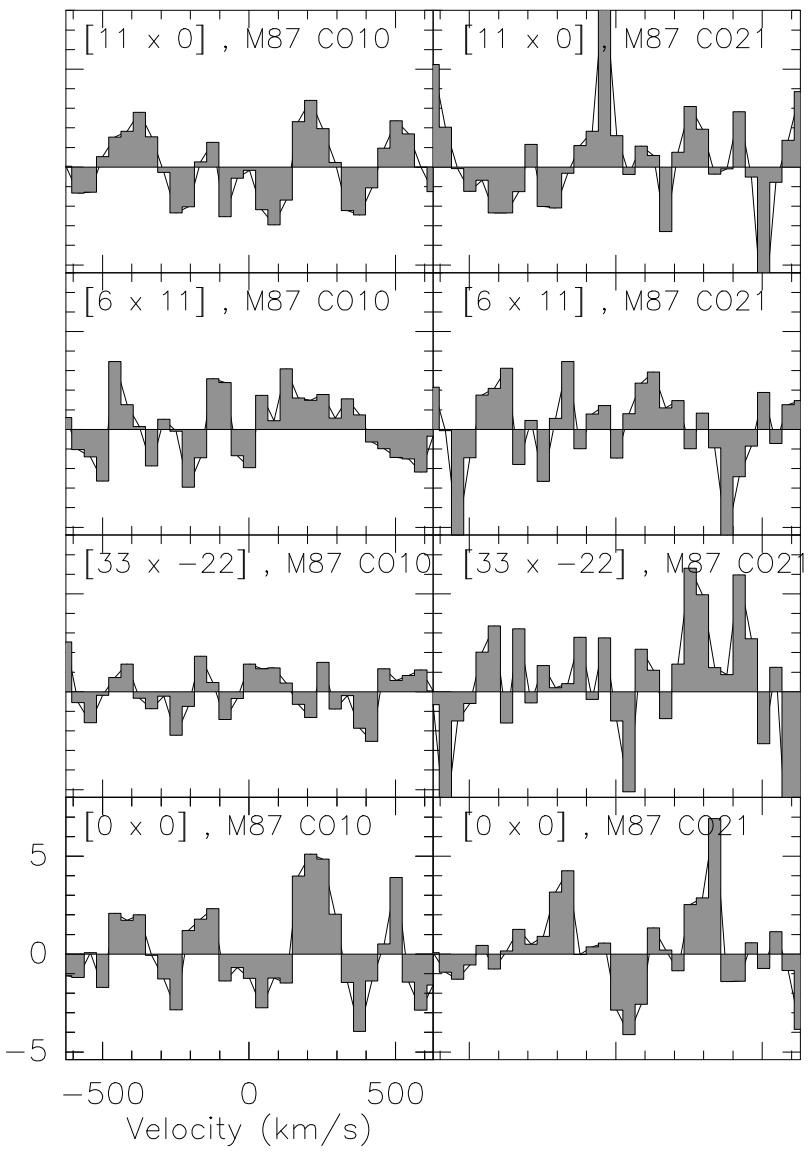

Fig. 4. M87 - $\mathrm{CO}(1-0)$ and $\mathrm{CO}(2-1)$ spectra obtained at all the positions observed as indicated at upper right in each diagram and as overlaid on Fig. 2. The channel width is $41.8 \mathrm{~km} \mathrm{~s}^{-1}$. See Table 2 for a summary. The $Y$-axis is in $T_{\mathrm{mb}}(\mathrm{mK})$.

volume, indicate that the activity cycle of the AGN in M87 is roughly $10^{8} \mathrm{yr}$ (Kaiser 2003). The largest radio structure is likely to be the remnant of an old bubble (Mathews \& Brighenti 2008). X-ray observations show a $30 \mathrm{kpc}$-long nearly radial filament of relatively cooler gas, aligned in projection with the radio lobe.

We have observed 4 points aligned along the radio lobes and $\mathrm{H} \alpha$ nebulosities (see Fig. 2). Only upper limits are found (see Table 2). The center of the galaxy is also not detected, see also NGC 4486 in Combes et al. (2007). This suggests that the feedback heating is very likely to be efficient enough to suppress the cooling, otherwise we should be able to observe cold gas falling back towards the center.

\section{Discussion and conclusions}

We observed 7 different positions along the radio lobes of MS0735.6+7421 and 4 positions for M87, in $\mathrm{CO}(1-0)$ with the $30 \mathrm{~m}$ telescope. We do not detect cold molecular gas in any of the observed regions. Our upper limits put significant constraints on the cold gas masses. We find $M_{\text {gas }} \leq$ a few $10^{9} M_{\odot}$ in MS0735.6+7421 and $M_{\text {gas }} \leq$ a few $10^{6} M_{\odot}$ in M87. The absence of cold gas could be explained if strong feedback heats sufficiently the intra-cluster medium and prevents any cooling. Nevertheless, as suggested by the recent results of high resolution CO mapping of NGC 1275 (Salomé et al. 2008), if the cooling happens only in very thin and elongated filaments, we may have missed such emission in MS0735.6+7421. The spatial and spectral dilution of a collection of CO filaments would make the detection difficult in a single beam of $28^{\prime \prime}(97 \mathrm{kpc})$ at this distance. More sensitive observations, with higher spatial resolution are needed to determine whether this is the case.

Acknowledgements. This work is based on observations done with the IRAM$30 \mathrm{~m}$ telescope. IRAM is supported by INSU/CNRS (France), MPG (Germany) and IGN (Spain). The authors thank the IRAM interferometer staff for help during the observations.

\section{References}

Combes, F., Young, L. M., \& Bureau, M. 2007, MNRAS, 377, 1795 Donahue, M., Stocke, J. T., \& Gioia, I. M. 1992, ApJ, 385, 49 Downes, D., \& Solomon, P. M. 1998, ApJ, 507, 615

Edge, A. C. 2001, MNRAS, 328, 762

Forman, W., Nulsen, P., Heinz, S., et al. 2005, ApJ, 635, 894

Forman, W., Jones, C., Churazov, E., et al. 2007, ApJ, 665, 1057

Ghizzardi, S., Molendi, S., Pizzolato, F., \& De Grandi, S. 2004, ApJ, 609, 638 Gitti, M., McNamara, B. R., Nulsen, P. E. J., \& Wise, M. W. 2007, ApJ, 660, 1118

Kaiser, C. R. 2003, MNRAS, 343, 1319

Mathews, W. G., \& Brighenti, F. 2008, ApJ, 676, 880

McNamara, B. R., \& Nulsen, P. E. J. 2007, ARA\&A, 45, 117

McNamara, B. R., Nulsen, P. E. J., Wise, M. W., et al. 2005, Nature, 433, 45

Revaz, Y., Combes, F., \& Salomé, P. 2008, A\&A, 477, L33

Salomé, P., \& Combes, F. 2003, A\&A, 412, 657

Salomé, P., \& Combes, F. 2004, A\&A, 415, L1

Salomé, P., Revaz, Y., Combes, F., et al. 2008, A\&A, 483, 793

Simionescu, A., Böhringer, H., Brüggen, M., \& Finoguenov, A. 2007, A\&A, 465,749

Solomon, P. M., Downes, D., Radford, S. J. E., \& Barrett, J. W. 1997, ApJ, 478, 144

Sparks, W. B., Ford, H. C., \& Kinney, A. L. 1993, ApJ, 413, 531

Young, A. J., Wilson, A. S., \& Mundell, C. G. 2002, ApJ, 579, 560 